\title{
On the Stability of Alternative Additive Equations in Multi- $\beta$-Normed Spaces
}

\author{
Xiuzhong Yang, Jing Ma, and Guofen Liu \\ College of Mathematics and Information Science, Hebei Normal University and Hebei Key Laboratory of \\ Computational Mathematics and Applications, Shijiazhuang 050024, China
}

Correspondence should be addressed to Guofen Liu; liugf2003@163.com

Received 7 April 2016; Accepted 30 June 2016

Academic Editor: Rudolf L. Stens

Copyright (C) 2016 Xiuzhong Yang et al. This is an open access article distributed under the Creative Commons Attribution License, which permits unrestricted use, distribution, and reproduction in any medium, provided the original work is properly cited.

We introduce the notion of multi- $\beta$-normed space $(0<\beta \leq 1)$ and study the stability of the alternative additive functional equation of two forms in this type of space.

\section{Introduction}

In 1940, Ulam [1] proposed the following stability problem: given a metric group $G(\cdot, \rho)$, a number $\varepsilon>0$, and mapping $f: G \rightarrow G$ which satisfies the inequality $\rho(f(x \cdot y), f(x)$. $f(y))<\varepsilon$ for all $x, y$ in $G$, does there exist an automorphism $a$ of $G$ and a constant $k>0$, depending only on $G$, such that $\rho(a(x), f(x)) \leq k \varepsilon$ for all $x$ in $G$ ? If the answer is affirmative, we call the equation $a(x \cdot y)=a(x) \cdot a(y)$ of automorphism stable. One year later, Hyers [2] provided a positive partial answer to Ulam's problem. In 1978, a generalized version of Hyers' result was proved by Rassias in [3]. Since then, the stability problems of several functional equations have been extensively investigated by a number of authors [4-12]. In particular, we also refer the readers to the survey paper [13] for recent developments in Ulam's type stability, [14] for recent developments of the conditional stability of the homomorphism equation, and books [15-18] for the general understanding of the stability theory.

The notion of multinormed space was introduced by Dales and Polyakov [19]. This concept is somewhat similar to operator sequence space and has some connections with operator spaces. Because of its applications in and outside of mathematics, the study on the stability of various functional equations has become one of the most important research subjects in the field of functional equations and attracts much attention from many researchers worldwide. Many examples of multinormed spaces can be found in [19], and further development of the stability in multinormed spaces can be found in papers [20-24].

In order to study the stability problem in more general setting, in this paper we introduce the notion of multi- $\beta$ normed spaces which are the combination of multinormed spaces and $\beta$-normed spaces, and the definition is given as follows.

In this paper we will use the following notations. Let $(E,\|\cdot\|)$ be a complex $\beta$-normed space with $0<\beta \leq 1$, and let $k \in N$. We denote by $E^{k}$ the linear space $E \oplus \cdots \oplus E$ consisting of $k$-tuples $\left(x_{1}, \ldots, x_{k}\right)$, where $x_{1}, \ldots, x_{k} \in E$. The linear operations on $E^{k}$ are defined coordinatewise. The zero element of either $E$ or $E^{k}$ is denoted by 0 . We denote the set $N_{k}=\{1,2, \ldots, k\}$ and denote by $S_{k}$ the group of permutation on $N_{k}$.

Definition 1. A multi- $\beta$-norm on $\left\{E^{k}: k \in N\right\}$ is a sequence $\left(\|\cdot\|_{k}\right)=\left(\|\cdot\|_{k}: k \in N\right)$ such that $\|\cdot\|_{k}$ is $\beta$-norm on $E^{k}$ for each $k \in N,\|x\|_{1}=\|x\|$ for each $x \in E$, and the following axioms are satisfied for each $k \in N$ with $k \geq 2$ :

$(A 1)\left\|\left(x_{\sigma(1)}, \ldots, x_{\sigma(k)}\right)\right\|_{k}=\left\|\left(x_{1}, \ldots, x_{k}\right)\right\|_{k},\left(\sigma \in S_{k}, x_{1}\right.$, $\left.\ldots, x_{k} \in E\right)$

(A2) $\left\|\left(\alpha_{1} x_{1}, \ldots, \alpha_{k} x_{k}\right)\right\|_{k} \leq\left(\max _{i \in N_{k}}\left|\alpha_{i}\right|^{\beta}\right)\left\|\left(x_{1}, \ldots, x_{k}\right)\right\|_{k}$, $\left(\alpha_{1}, \ldots, \alpha_{k} \in C, x_{1}, \ldots, x_{k} \in E\right)$;

(A3) $\left\|\left(x_{1}, \ldots, x_{k-1}, 0\right)\right\|_{k}=\left\|\left(x_{1}, \ldots, x_{k-1}\right)\right\|_{k-1},\left(x_{1}, \ldots\right.$, $\left.x_{k} \in E\right)$ 
(A4) $\left\|\left(x_{1}, \ldots, x_{k-1}, x_{k-1}\right)\right\|_{k}=\left\|\left(x_{1}, \ldots, x_{k-1}\right)\right\|_{k-1},\left(x_{1}, \ldots\right.$, $\left.x_{k} \in E\right)$.

In this case, we say that $\left(\left(E^{k},\|\cdot\|_{k}\right): k \in N\right)$ is a multi- $\beta$ normed space.

The following two properties of multi- $\beta$-normed spaces are easily obtained:

$$
\begin{aligned}
\|(x, \ldots, x)\|_{k} & =\|x\|, \quad(x \in E) ; \\
\max _{i \in N_{k}}\left\|x_{i}\right\| & \leq\left\|\left(x_{1}, \ldots, x_{k}\right)\right\|_{k} \leq \sum_{i=1}^{k}\left\|x_{i}\right\| \\
& \leq k \max _{i \in N_{k}}\left\|x_{i}\right\|, \quad\left(x_{1}, \ldots, x_{k} \in E\right) .
\end{aligned}
$$

It follows from $(2)$ that if $(E,\|\cdot\|)$ is a complete $\beta$-normed space, then $\left(E^{k},\|\cdot\|_{k}\right)$ is complete $\beta$-normed space for each $k \in N$; in this case $\left(\left(E^{k},\|\cdot\|_{k}\right): k \in N\right)$ is a complete multi$\beta$-normed space. In particular, if $\beta=1(E,\|\cdot\|)$ is Banach space, then the space $\left(\left(E^{k},\|\cdot\|_{k}\right): k \in N\right)$ is multi-Banach space. Now we give one example of multi- $\beta$-normed space.

Example 2. Let $E$ be an arbitrary $\beta$-normed space. The sequence $\left(\|\cdot\|_{k}, k \in N\right)$ on $E^{k}: k \in N$ defined by

$$
\left\|\left(x_{1}, \ldots, x_{k}\right)\right\|_{k}=\max _{i \in N_{k}}\left\|x_{i}\right\|, \quad\left(x_{1}, \ldots, x_{k} \in E\right),
$$

is a multi- $\beta$-norm.

Lemma 3. Let $k \in N$ and $\left(x_{1}, \ldots, x_{k}\right) \in E^{k}$. For each $j \in\{1, \ldots, k\}$, let $\left(x_{n}^{j}\right)_{n=1,2, \ldots}$ be a sequence in $E$ such that $\lim _{n \rightarrow \infty} x_{n}^{j}=x_{j}$. Then for each $\left(y_{1}, \ldots, y_{k}\right) \in E^{k}$ one has

$$
\lim _{n \rightarrow \infty}\left(x_{n}^{1}-y_{1}, \ldots, x_{n}^{k}-y_{k}\right)=\left(x_{1}-y_{1}, \ldots, x_{k}-y_{k}\right) \text {. }
$$

Definition 4. Let $\left(\left(E^{k},\|\cdot\|_{k}\right): k \in N\right)$ be a multi- $\beta$-normed space. A sequence $\left(x_{n}\right)$ in $E$ is a multinull sequence if, for each $\varepsilon>0$, there exists $n_{0} \in N$ such that

$$
\sup _{k \in N}\left\|\left(x_{n}, \ldots, x_{n+k-1}\right)\right\|_{k}<\varepsilon
$$

for all $n \geq n_{0}$. Let $x \in E$; we say that the sequence $\left(x_{n}\right)$ is multiconvergent to $x$ in $E$ if $\left(x_{n}-x\right)$ is a multinull sequence. In this case, $x$ is called the limit of the sequence $\left(x_{n}\right)$ and we denote it by $\lim _{n \rightarrow \infty} x_{n}=x$.

In this paper we will study the stability in the multi$\beta$-normed space of alternative additive equation of the two forms, which were further studied in the normed spaces in paper [25], and their definitions are presented as follows.

Definition 5 (see [25]). Let $X, Y$ be linear spaces and let $A$ be mapping from $X$ to $Y$. The equation is called alternative additive of the first form if $A$ satisfies the functional equation

$$
A\left(x_{1}+x_{2}\right)+A\left(x_{1}-x_{2}\right)=-2 A\left(-x_{1}\right) .
$$

Obviously (6) is equivalent to the alternative Jensen equation

$$
A\left(-\frac{x+y}{2}\right)=-\frac{1}{2}[A(x)+A(y)] .
$$

Definition 6 (see [25]). Let $X, Y$ be linear spaces and let $A$ be mapping from $X$ to $Y$. The equation is called alternative additive of the second form if $A$ satisfies the functional equation

$$
A\left(x_{1}+x_{2}\right)+A\left(x_{1}-x_{2}\right)=-2 A\left(-x_{2}\right) .
$$

Obviously (6) is equivalent to the alternative Jensen equation

$$
A\left(-\frac{x-y}{2}\right)=-\frac{1}{2}[A(x)-A(y)] .
$$

\section{Stability of Alternative Additive Equation of the First Form}

In this section we will study the stability of the alternative additive equation of the first form in multi- $\beta$-normed space and on the restricted domain. First, we investigate the general case where the domain of the mapping is the whole space. The following theorem is obtained.

Theorem 7. Let $X$ be a real normed space, and let $\left(\left(Y^{n},\|\cdot\|\right)\right.$ : $n \in N)$ be a complete real multi- $\beta$-normed space. Suppose that $\delta \geq 0$; mapping $f: X \rightarrow Y$ satisfies

$$
\begin{aligned}
& \sup _{k \in N} \|\left(f\left(x_{1}+y_{1}\right)+f\left(x_{1}-y_{1}\right)\right. \\
& \quad+2 f\left(-x_{1}\right), \ldots, f\left(x_{k}+y_{k}\right)+f\left(x_{k}-y_{k}\right) \\
& \left.\quad+2 f\left(-x_{k}\right)\right) \|_{k} \leq \delta ; \\
& \sup _{k \in N}\left\|\left(f\left(-z_{1}\right)+f\left(z_{1}\right), \ldots, f\left(-z_{k}\right)+f\left(z_{k}\right)\right)\right\|_{k} \leq \frac{\delta}{2^{\beta}}
\end{aligned}
$$

for all $x_{1}, \ldots, x_{k}, y_{1}, \ldots, y_{k}, z_{1}, \ldots, z_{k} \in X$. Then there exists unique alternative additive mapping of the first form $A: X \rightarrow$ $Y$ satisfying

$$
\begin{aligned}
\sup _{k \in N} & \left\|\left(f\left(z_{1}\right)-A\left(z_{1}\right), \ldots, f\left(z_{k}\right)-A\left(z_{k}\right)\right)\right\|_{k} \\
\leq & \left(2 \delta+\frac{\delta}{4^{\beta}}\right) \cdot \frac{1}{2^{\beta}-1}
\end{aligned}
$$

for all $z_{1}, \ldots, z_{k} \in X$.

Proof. Letting $x_{i}=y_{i}=0(i=1, \ldots, k)$ in (10) yields

$$
\sup _{k \in N}\|(f(0), \ldots, f(0))\|_{k} \leq \frac{\delta}{4^{\beta}} \text {. }
$$

Setting $x_{i}=y_{i}=z_{i}(i=1, \ldots, k)$ yields

$$
\begin{aligned}
& \sup _{k \in N} \|\left(f\left(2 z_{1}\right)+f(0)+2 f\left(-z_{1}\right), \ldots, f\left(2 z_{k}\right)+f(0)\right. \\
& \left.\quad+2 f\left(-z_{k}\right)\right) \|_{k} \leq \delta .
\end{aligned}
$$


It follows from (11), (13), and (14) that

$$
\begin{aligned}
& \sup _{k \in N}\left\|\left(f\left(2 z_{1}\right)-2 f\left(z_{1}\right), \ldots, f\left(2 z_{k}\right)-2 f\left(z_{k}\right)\right)\right\|_{k} \\
& \quad \leq \sup _{k \in N} \|\left(f\left(2 z_{1}\right)+f(0)+2 f\left(-z_{1}\right), \ldots, f\left(2 z_{k}\right)\right. \\
&\left.\quad+f(0)+2 f\left(-z_{k}\right)\right) \|_{k} \\
& \quad+\sup _{k \in N} \|\left(-2\left(f\left(-z_{1}\right)+f\left(z_{1}\right)\right), \ldots,\right. \\
&\left.\quad-2\left(f\left(-z_{k}\right)+f\left(z_{k}\right)\right)\right)\left\|_{k}+\sup _{k \in N}\right\|(-f(0), \ldots, \\
&\quad-f(0)) \|_{k} \leq 2 \delta+\frac{\delta}{4^{\beta}} .
\end{aligned}
$$

Therefore, we have

$$
\begin{gathered}
\sup _{k \in N} \|\left(\frac{1}{2^{n}} f\left(2^{n} z_{1}\right)-f\left(z_{1}\right), \ldots, \frac{1}{2^{n}} f\left(2^{n} z_{k}\right)\right. \\
\left.-f\left(z_{k}\right)\right) \|_{k} \leq\left(2 \delta+\frac{\delta}{4^{\beta}}\right) \sum_{k=1}^{n} \frac{1}{2^{k \beta}}, \\
\sup _{k \in N} \|\left(\frac{1}{2^{n+m}} f\left(2^{n+m} z_{1}\right)\right. \\
-\frac{1}{2^{n}} f\left(2^{n} z_{1}\right), \ldots, \frac{1}{2^{n+m}} f\left(2^{n+m} z_{k}\right) \\
\left.-\frac{1}{2^{n}} f\left(2^{n} z_{k}\right)\right) \|_{k} \leq\left(2 \delta+\frac{\delta}{4^{\beta}}\right) \sum_{k=n+1}^{n+m} \frac{1}{2^{k \beta}}
\end{gathered}
$$

for all $m, n \in N, m \geq 1$.

It follows from $(A 2)$ and (17) that

$$
\begin{aligned}
\sup _{k \in N} & \|\left(\frac{1}{2^{n+m}} f\left(2^{n+m} x\right)-\frac{1}{2^{n}} f\left(2^{n} x\right), \ldots, \frac{1}{2^{n+m+k-1}}\right. \\
\cdot & \left.f\left(2^{n+m+k-1} x\right)-\frac{1}{2^{n+k-1}} f\left(2^{n+k-1} x\right)\right) \|_{k} \\
& =\sup _{k \in N} \|\left(\frac{1}{2^{n+m}} f\left(2^{n+m} x\right)-\frac{1}{2^{n}} f\left(2^{n} x\right), \ldots,\right. \\
& \frac{1}{2^{k-1}}\left(\frac{1}{2^{n+m}} f\left(2^{n+m}\left(2^{k-1} x\right)\right)\right. \\
& \left.\left.-\frac{1}{2^{n}} f\left(2^{n}\left(2^{k-1} x\right)\right)\right)\right)\left\|_{k} \leq \sup _{k \in N}\right\|\left(\frac{1}{2^{n+m}} f\left(2^{n+m} x\right)\right. \\
& -\frac{1}{2^{n}} f\left(2^{n} x\right), \ldots, \frac{1}{2^{n+m}} f\left(2^{n+m}\left(2^{k-1} x\right)\right)-\frac{1}{2^{n}} \\
& \left.\cdot f\left(2^{n}\left(2^{k-1} x\right)\right)\right) \|_{k} \leq\left(2 \delta+\frac{\delta}{4^{\beta}}\right) \sum_{k=n+1}^{n+m} \frac{1}{2^{k \beta}} .
\end{aligned}
$$

Hence $\left\{\left(1 / 2^{n}\right) f\left(2^{n} x\right)\right\}$ is Cauchy sequence, which must be convergent in complete real multi- $\beta$-normed space; that is, there exists mapping $A: X \rightarrow Y$ such that $A(x)$ := $\lim _{n \rightarrow \infty}\left(1 / 2^{n}\right) f\left(2^{n} x\right)$. Hence, for arbitrary $\varepsilon>0$, there exists $n_{0} \in N$; if $n \geq n_{0}$, then we have

$$
\begin{gathered}
\sup _{k \in N} \|\left(\frac{1}{2^{n}} f\left(2^{n} x\right)-A(x), \ldots, \frac{1}{2^{n+k-1}} f\left(2^{n+k-1} x\right)\right. \\
-A(x)) \|_{k}<\varepsilon .
\end{gathered}
$$

Considering (2), we obtain

$$
\lim _{n \rightarrow \infty}\left\|\frac{1}{2^{n}} f\left(2^{n} x\right)-A(x)\right\|=0, \quad x \in X .
$$

If we let $n=0$ in (17), then we have

$$
\begin{gathered}
\sup _{k \in N} \|\left(\frac{1}{2^{m}} f\left(2^{m} z_{1}\right)-f\left(z_{1}\right), \ldots, \frac{1}{2^{m}} f\left(2^{m} z_{k}\right)\right. \\
\left.-f\left(z_{k}\right)\right) \|_{k} \leq\left(2 \delta+\frac{\delta}{4^{\beta}}\right) \sum_{k=1}^{m} \frac{1}{2^{k \beta}} .
\end{gathered}
$$

Letting $m \rightarrow \infty$ and making use of Lemma 3 and (20), we know that mapping $A$ satisfies (12).

Let $x, y \in X$. Setting $x_{1}=\cdots=x_{k}=2^{n} x, y_{1}=\cdots=y_{k}=$ $2^{n} y$ in (10) and dividing both sides by $2^{n \beta}$ yield

$$
\begin{aligned}
\sup _{k \in N} & \|\left(\frac{1}{2^{n}} f\left(2^{n}(x+y)\right)+\frac{1}{2^{n}} f\left(2^{n}(x-y)\right)+2\right. \\
\cdot & \frac{1}{2^{n}} f\left(-2^{n} x\right), \ldots, \frac{1}{2^{n}} f\left(2^{n}(x+y)\right) \\
+ & \left.\frac{1}{2^{n}} f\left(2^{n}(x-y)\right)+2 \cdot \frac{1}{2^{n}} f\left(-2^{n} x\right)\right) \|_{k} \leq \frac{\delta}{2^{n \beta}},
\end{aligned}
$$

which together with (1) implies

$$
\begin{gathered}
\| \frac{1}{2^{n}} f\left(2^{n}(x+y)\right)+\frac{1}{2^{n}} f\left(2^{n}(x-y)\right)+2 \\
\cdot \frac{1}{2^{n}} f\left(-2^{n} x\right) \| \leq \frac{\delta}{2^{n \beta}} .
\end{gathered}
$$

Taking limit as $n \rightarrow \infty$, we have

$$
A(x+y)+A(x-y)+2 A(-x)=0, \quad(x, y \in X) .
$$

So $A$ is the alternative additive mapping of the first form. It remains to show that $A$ is uniquely determined. Let $A^{\prime}: X \rightarrow$ $Y$ be another alternative additive mapping of the first form that satisfies (12). It follows from (24) that some properties of mapping $A$ are obtained:

(1) If we let $y=0$, we get $A(x)=-A(x)$, so $A$ is odd mapping.

(2) If we let $x=y=0$, we have $A(0)=0$.

(3) Putting $y=x$ yields $A(2 x)=2 A(x)$; that is, $A(x)=$ $(1 / 2) A(2 x)$.

(4) Replacing $x, y$ with $2 x$, respectively, yields $A\left(2^{2} x\right)=$ $2^{2} A(x)$; hence $A(x)=\left(1 / 2^{2}\right) A\left(2^{2} x\right)$. 
(5) Replacing $x, y$ with $2^{2} x$, respectively, yields $A\left(2^{3} x\right)=$ $2^{3} A(x)$; that is, $A(x)=\left(1 / 2^{3}\right) A\left(2^{3} x\right)$.

Proceeding in an obvious fashion yields $A(x)=$ $\left(1 / 2^{n}\right) A\left(2^{n} x\right)$. Similarly, we have $A^{\prime}(x)=\left(1 / 2^{n}\right) A^{\prime}\left(2^{n} x\right)$. Letting $z_{1}=\cdots=z_{k}=x$ in (12) and in view of (1) we obtain

$$
\|f(x)-A(x)\| \leq\left(2 \delta+\frac{\delta}{4^{\beta}}\right) \cdot \frac{1}{2^{\beta}-1} .
$$

Similarly we have

$$
\left\|f(x)-A^{\prime}(x)\right\| \leq\left(2 \delta+\frac{\delta}{4^{\beta}}\right) \cdot \frac{1}{2^{\beta}-1} .
$$

Therefore,

$$
\begin{aligned}
\left\|A(x)-A^{\prime}(x)\right\|= & \left\|\frac{1}{2^{n}} A\left(2^{n} x\right)-\frac{1}{2^{n}} A^{\prime}\left(2^{n} x\right)\right\| \\
\leq & \frac{1}{2^{n \beta}}\left\|A\left(2^{n} x\right)-A^{\prime}\left(2^{n} x\right)\right\| \\
\leq & \frac{1}{2^{n \beta}}\left\|A\left(2^{n} x\right)-f\left(2^{n} x\right)\right\| \\
& +\frac{1}{2^{n \beta}}\left\|f\left(2^{n} x\right)-A^{\prime}\left(2^{n} x\right)\right\| \\
\leq & \frac{1}{2^{n \beta}}\left(2 \delta+\frac{\delta}{4^{\beta}}\right) \cdot \frac{2}{2^{\beta}-1} .
\end{aligned}
$$

Taking limit as $n \rightarrow \infty$, we have $A^{\prime}=A$.

It is a time to study the stability of this type mapping on the local domain. We only prove the stability result when the target spaces are real multi-Banach spaces, that is, the special case of real multi- $\beta$-normed space when $\beta=1$. For $0<\beta<1$, it is an interesting open problem. The following are our results.

Theorem 8. Let $X$ be a real normed space, let $\left(\left(Y^{n},\|\cdot\|\right): n \in\right.$ $N)$ be a real multi-Banach space, and let $d>0, \delta \geq 0$. Suppose that mapping $f: X \rightarrow Y$ satisfies

$$
\begin{aligned}
& \|\left(f\left(x_{1}+y_{1}\right)+f\left(x_{1}-y_{1}\right)\right. \\
& \quad+2 f\left(-x_{1}\right), \ldots, f\left(x_{k}+y_{k}\right)+f\left(x_{k}-y_{k}\right) \\
& \left.\quad+2 f\left(-x_{k}\right)\right) \|_{k} \leq \delta, \\
& \left\|\left(f\left(z_{1}\right)+f\left(-z_{1}\right), \ldots, f\left(z_{k}\right)+f\left(-z_{k}\right)\right)\right\|_{k} \leq \frac{\delta}{2}
\end{aligned}
$$

for all $x_{1}, \ldots, x_{k}, y_{1}, \ldots, y_{k}, z_{1}, \ldots, z_{k} \in X$ that satisfy $\left\|\left(x_{1}, \ldots, x_{k}\right)\right\|_{k}+\left\|\left(y_{1}, \ldots, y_{k}\right)\right\|_{k} \geq d$ and $\left\|\left(z_{1}, \ldots, z_{k}\right)\right\|_{k} \geq d$. Then there exists unique alternative additive mapping of the first form $A: X \rightarrow Y$ such that

$$
\sup _{k \in N}\left\|\left(f\left(z_{1}\right)-A\left(z_{1}\right), \ldots, f\left(z_{k}\right)-A\left(z_{k}\right)\right)\right\|_{k} \leq \frac{95}{4} \delta
$$

for all $z_{1}, \ldots, z_{k} \in X$.
Proof. Fix $k \in N$. Let $\mathbf{X}=\left(x_{1}, \ldots, x_{k}\right)$ and $\mathbf{Y}=\left(y_{1}, \ldots, y_{k}\right)$ satisfy $\left\|\left(x_{1}, \ldots, x_{k}\right)\right\|_{k}+\left\|\left(y_{1}, \ldots, y_{k}\right)\right\|_{k}<d$. If $\mathbf{X}=\mathbf{Y}=\mathbf{0}$, then let $\mathbf{T}=\left(t_{1}, \ldots, t_{k}\right) \in X^{k}$ and $\|\mathbf{T}\|_{k}=d$. If $\mathbf{X} \neq \mathbf{0}$ or $\mathbf{Y} \neq \mathbf{0}$, let

$$
\mathbf{T}= \begin{cases}\left(1+\frac{d}{\|\mathbf{X}\|_{k}}\right) \mathbf{X}, & \|\mathbf{X}\|_{k} \geq\|\mathbf{Y}\|_{k} \\ \left(1+\frac{d}{\|\mathbf{Y}\|_{k}}\right) \mathbf{Y}, & \|\mathbf{X}\|_{k}<\|\mathbf{Y}\|_{k}\end{cases}
$$

If $\|\mathbf{X}\|_{k} \geq\|\mathbf{Y}\|_{k}$, we get $\|\mathbf{T}\|_{k}=\|\mathbf{X}\|_{k}+d>d$. If $\|\mathbf{X}\|_{k}<\|\mathbf{Y}\|_{k}$, we have $\|\mathbf{T}\|_{k}=\|\mathbf{Y}\|_{k}+d>d$. Therefore,

$$
\begin{aligned}
&\|\mathbf{X}-\mathbf{T}\|_{k}+\|\mathbf{Y}+\mathbf{T}\|_{k} \geq 2\|\mathbf{T}\|_{k}-\left(\|\mathbf{X}\|_{k}+\|\mathbf{Y}\|_{k}\right) \geq d ; \\
&\|\mathbf{X}-\mathbf{T}\|_{k}+\|\mathbf{Y}-\mathbf{T}\|_{k} \geq 2\|\mathbf{T}\|_{k}-\left(\|\mathbf{X}\|_{k}+\|\mathbf{Y}\|_{k}\right) \geq d ; \\
&\|\mathbf{X}-2 \mathbf{T}\|_{k}+\|\mathbf{Y}\|_{k} \geq 2\|\mathbf{T}\|_{k}-\left(\|\mathbf{X}\|_{k}+\|\mathbf{Y}\|_{k}\right) \geq d ; \text { for }\|\mathbf{X}\|_{k} \geq\|\mathbf{Y}\|_{k} ; \\
&\|\mathbf{X} \pm \mathbf{T}\|_{k} \geq\|\mathbf{T}\|_{k}-\|\mathbf{X}\|_{k}=\left(\|\mathbf{X}\|_{k}+d\right)-\|\mathbf{X}\|_{k}=d, \\
&\|\mathbf{X} \pm \mathbf{T}\|_{k} \geq\|\mathbf{T}\|_{k}-\|\mathbf{X}\|_{k} \\
&=\left(\|\mathbf{Y}\|_{k}+d\right)-\|\mathbf{X}\|_{k}=d, \\
& \text { for }\|\mathbf{Y}\|_{k} \geq\|\mathbf{X}\|_{k} ;
\end{aligned}
$$

$$
\|\mathbf{T}-\mathbf{X}\|_{k}+\|\mathbf{T}\|_{k} \geq d .
$$

It follows from (28) that

$$
\begin{aligned}
& \|\left(f\left(x_{1}+y_{1}\right)+f\left(x_{1}-y_{1}\right)\right. \\
& +2 f\left(-x_{1}\right), \ldots, f\left(x_{k}+y_{k}\right)+f\left(x_{k}-y_{k}\right) \\
& \left.+2 f\left(-x_{k}\right)\right)\left\|_{k} \leq\right\|\left(f\left(x_{1}+y_{1}\right)\right. \\
& + \\
& + \\
& +2 f\left(x_{1}-y_{1}-2 t_{1}\right) \\
& + \\
& + \\
& \left.+\|\left(x_{1}-t_{1}\right)\right), \ldots, f\left(x_{k}+y_{k}\right) \\
& \left.\left.+2 f\left(-x_{k}\right)+2 f\left(-\left(x_{1}-t_{1}\right)\right), \ldots, f\left(x_{1}\right)\right)\right) \|_{k} \\
& \left.-f\left(x_{k}-y_{k}\right)+2 f\left(-\left(x_{k}-t_{k}\right)\right)\right) \|_{k} \\
& +\|\left(f\left(x_{1}+y_{1}-2 t_{1}\right)+f\left(x_{1}-y_{1}-2 t_{1}\right)\right. \\
& +2 f\left(-\left(x_{1}-2 t_{1}\right)\right), \ldots, f\left(x_{k}+y_{k}-2 t_{k}\right) \\
& + \\
& \left.+f\left(x_{k}-y_{k}-2 t_{k}\right)+2 f\left(-\left(x_{k}-2 t_{k}\right)\right)\right) \|_{k} \\
& +\|\left(2 f\left(\left(t_{1}-x_{1}\right)+t_{1}\right)+2 f\left(-x_{1}\right)\right. \\
& +4 f\left(x_{1}-t_{1}\right), \ldots, 2 f\left(\left(t_{k}-x_{k}\right)+t_{k}\right)+2 f\left(-x_{k}\right) \\
& \left.+4 f\left(x_{k}-t_{k}\right)\right)\left\|_{k}+\right\|\left(4 f\left(x_{1}-t_{1}\right)\right.
\end{aligned}
$$




$$
\begin{aligned}
& \quad+4 f\left(-\left(x_{1}-t_{1}\right)\right), \ldots, 4 f\left(x_{k}-t_{k}\right) \\
& \left.+4 f\left(-\left(x_{k}-t_{k}\right)\right)\right) \|_{k} \leq 7 \delta, \\
& \left\|\left(2 f\left(z_{1}\right)+2 f\left(-z_{1}\right), \ldots, 2 f\left(z_{k}\right)+2 f\left(-z_{k}\right)\right)\right\|_{k} \\
& \quad \leq \|\left(2 f\left(z_{1}\right)+f\left(-z_{1}+t_{1}\right)\right. \\
& +f\left(-z_{1}-t_{1}\right), \ldots, 2 f\left(z_{k}\right)+f\left(-z_{k}+t_{k}\right) \\
& \left.+f\left(-z_{k}-t_{k}\right)\right)\left\|_{k}+\right\|\left(-f\left(-z_{1}-t_{1}\right)\right. \\
& \left.-f\left(z_{1}+t_{1}\right), \ldots,-f\left(-z_{k}-t_{k}\right)-f\left(z_{k}+t_{k}\right)\right) \|_{k} \\
& +\|\left(-f\left(z_{1}-t_{1}\right)-f\left(-z_{1}+t_{1}\right), \ldots,-f\left(z_{k}-t_{k}\right)\right. \\
& \left.-f\left(-z_{k}+t_{k}\right)\right)\left\|_{k}+\right\|\left(2 f\left(-z_{1}\right)+f\left(z_{1}-t_{1}\right)\right. \\
& +f\left(z_{1}+t_{1}\right), \ldots, 2 f\left(-z_{k}\right)+f\left(z_{k}-t_{k}\right) \\
& \left.+f\left(z_{k}+t_{k}\right)\right) \|_{k} \leq 15 \delta .
\end{aligned}
$$

It follows from Theorem 7 that there exists unique alternative additive mapping of the first form $A: X \rightarrow Y$ satisfying (29) for all $z_{1}, \ldots, z_{k} \in X$.

Corollary 9. Let $\left(\left(X^{n},\|\cdot\|\right): n \in N\right)$ be a real multinormed space, and let $\left(\left(Y^{n},\|\cdot\|\right): n \in N\right)$ be a multi-Banach space. Mapping $f: X \rightarrow Y$ satisfies alternative additive equation of the first form if and only if, for each $k \in N$, if $\left\|\left(x_{1}, \ldots, x_{k}\right)\right\|_{k}+$ $\left\|\left(y_{1}, \ldots, y_{k}\right)\right\|_{k} \rightarrow \infty$ and $\left\|\left(z_{1}, \ldots, z_{k}\right)\right\|_{k} \rightarrow \infty$, one has

$$
\begin{aligned}
& \|\left(f\left(x_{1}+y_{1}\right)+f\left(x_{1}-y_{1}\right)\right. \\
& \quad+2 f\left(-x_{1}\right), \ldots, f\left(x_{k}+y_{k}\right)+f\left(x_{k}-y_{k}\right) \\
& \left.\quad+2 f\left(-x_{k}\right)\right) \|_{k} \longrightarrow 0 ; \\
& \left\|\left(f\left(z_{1}\right)+f\left(-z_{1}\right), \ldots, f\left(z_{k}\right)+f\left(-z_{k}\right)\right)\right\|_{k} \longrightarrow 0 .
\end{aligned}
$$

\section{Stability of Alternative Additive Equation of the Second Form}

In this section we will study the stability of the alternative additive equation of the second form in multi- $\beta$-normed space and on the restricted domain. First, we investigate the general case where the domain of the mapping is the whole space. The following theorem is obtained.

Theorem 10. Let $X$ be a real normed space, and let $\left(\left(Y^{n},\|\cdot\|\right)\right.$ : $n \in N)$ be a complete real multi- $\beta$-normed space. Suppose that $\delta \geq 0$; mapping $f: X \rightarrow Y$ satisfies

$$
\begin{aligned}
& \sup _{k \in N} \|\left(f\left(x_{1}+y_{1}\right)-f\left(x_{1}-y_{1}\right)\right. \\
& \quad+2 f\left(-y_{1}\right), \ldots, f\left(x_{k}+y_{k}\right)-f\left(x_{k}-y_{k}\right) \\
& \left.\quad+2 f\left(-y_{k}\right)\right) \|_{k} \leq \delta
\end{aligned}
$$

for all $x_{1}, \ldots, x_{k}, y_{1}, \ldots, y_{k} \in X$. Then there exists unique alternative additive mapping of the second form $A: X \rightarrow Y$ such that

$$
\begin{gathered}
\sup _{k \in N}\left\|\left(f\left(x_{1}\right)-A\left(x_{1}\right), \ldots, f\left(x_{k}\right)-A\left(x_{k}\right)\right)\right\|_{k} \\
\leq \frac{2^{2 \beta}+2^{\beta}+1}{2^{\beta}\left(2^{\beta}-1\right)} \delta
\end{gathered}
$$

for all $x_{1}, \ldots, x_{k} \in X$.

Proof. Let $x_{i}=y_{i}=0(i=1, \ldots, k)$ in (34); we get

$$
\sup _{k \in N}\|(f(0), \ldots, f(0))\|_{k} \leq \frac{\delta}{2^{\beta}} .
$$

Replacing $y_{i}(i=1, \ldots, k)$ with $x_{i}$, we obtain

$$
\begin{aligned}
\sup _{k \in N} & \|\left(f\left(2 x_{1}\right)-f(0)+2 f\left(-x_{1}\right), \ldots, f\left(2 x_{k}\right)\right. \\
& \left.-f(0)+2 f\left(-x_{k}\right)\right) \|_{k} \leq \delta .
\end{aligned}
$$

Let $x_{1}=\cdots=x_{k}=0$ and replace $y_{i}(i=1, \ldots, k)$ with $x_{i}$; we obtain

$$
\sup _{k \in N}\left\|\left(f\left(x_{1}\right)+f\left(-x_{1}\right), \ldots, f\left(x_{k}\right)+f\left(-x_{k}\right)\right)\right\|_{k} \leq \delta .
$$

Hence for all $x_{1}, \ldots, x_{k} \in X$ we have

$$
\begin{aligned}
& \sup _{k \in N}\left\|\left(f\left(2 x_{1}\right)-2 f\left(x_{1}\right), \ldots, f\left(2 x_{k}\right)-2 f\left(x_{k}\right)\right)\right\|_{k} \\
& \quad \leq \sup _{k \in N} \|\left(f\left(2 x_{1}\right)-f(0)+2 f\left(-x_{1}\right), \ldots, f\left(2 x_{k}\right)\right. \\
& \left.\quad-f(0)+2 f\left(-x_{k}\right)\right) \|_{k} \\
& \quad+\sup _{k \in N} \|\left(2\left(f\left(x_{1}\right)+f\left(-x_{1}\right)\right), \ldots,\right. \\
& \left.\quad 2\left(f\left(x_{k}\right)+f\left(-x_{k}\right)\right)\right)\left\|_{k}+\sup _{k \in N}\right\|(f(0), \ldots, f(0)) \|_{k} \\
& \quad \leq \frac{2^{2 \beta}+2^{\beta}+1}{2^{\beta}} \delta .
\end{aligned}
$$

Therefore, for all $m, n \in N, m \geq 1$, we have

$$
\begin{aligned}
\sup _{k \in N} & \|\left(\frac{1}{2^{n}} f\left(2^{n} x_{1}\right)-f\left(x_{1}\right), \ldots, \frac{1}{2^{n}} f\left(2^{n} x_{k}\right)\right. \\
- & \left.f\left(x_{k}\right)\right) \|_{k} \leq\left(\frac{2^{2 \beta}+2^{\beta}+1}{2^{\beta}} \delta\right) \sum_{k=1}^{n} \frac{1}{2^{k \beta}}, \\
\sup _{k \in N} & \|\left(\frac{1}{2^{n+m}} f\left(2^{n+m} x_{1}\right)\right. \\
& -\frac{1}{2^{n}} f\left(2^{n} x_{1}\right), \ldots, \frac{1}{2^{n+m}} f\left(2^{n+m} x_{k}\right) \\
& \left.-\frac{1}{2^{n}} f\left(2^{n} x_{k}\right)\right) \|_{k} \leq\left(\frac{2^{2 \beta}+2^{\beta}+1}{2^{\beta}} \delta\right) \sum_{k=n+1}^{n+m} \frac{1}{2^{k \beta}} .
\end{aligned}
$$

We omit the following arguments because they are similar to that of Theorem 7. 
Theorem 11. Let $X$ be a real normed space, let $\left(\left(Y^{n},\|\cdot\|\right): n \in\right.$ $N)$ be a complete real multi-Banach space, and let $d>0, \delta \geq 0$. Suppose that $f: X \rightarrow Y$ satisfies

$$
\begin{aligned}
& \|\left(f\left(x_{1}+y_{1}\right)-f\left(x_{1}-y_{1}\right)\right. \\
& \quad+2 f\left(-y_{1}\right), \ldots, f\left(x_{k}+y_{k}\right)-f\left(x_{k}-y_{k}\right) \\
& \left.\quad+2 f\left(-y_{k}\right)\right) \|_{k} \leq \delta \\
& \left\|\left(f\left(z_{1}\right)+f\left(-z_{1}\right), \ldots, f\left(z_{k}\right)+f\left(-z_{k}\right)\right)\right\|_{k} \leq \frac{\delta}{2}
\end{aligned}
$$

for $x_{1}, \ldots, x_{k}, y_{1}, \ldots, y_{k}, z_{1}, \ldots, z_{k} \in X$ that satisfy $\|\left(x_{1}\right.$, $\left.\ldots, x_{k}\right)\left\|_{k}+\right\|\left(y_{1}, \ldots, y_{k}\right) \|_{k} \geq d$ and $\left\|\left(z_{1}, \ldots, z_{k}\right)\right\|_{k} \geq d$. Then there exists unique alternative additive mapping of the second form $A: X \rightarrow Y$ such that

$$
\sup _{k \in N}\left\|\left(f\left(x_{1}\right)-A\left(x_{1}\right), \ldots, f\left(x_{k}\right)-A\left(x_{k}\right)\right)\right\|_{k} \leq \frac{49}{2} \delta
$$

for all $x_{1}, \ldots, x_{k} \in X$.

Proof. Fix $k \in N$; choose $\mathbf{X}=\left(x_{1}, \ldots, x_{k}\right)$ and $\mathbf{Y}=\left(y_{1}\right.$, $\left.\ldots, y_{k}\right)$ with $\left\|\left(x_{1}, \ldots, x_{k}\right)\right\|_{k}+\left\|\left(y_{1}, \ldots, y_{k}\right)\right\|_{k}<d$. If $\mathbf{X}=\mathbf{Y}=$ $\mathbf{0}$, then let $\mathbf{T}=\left(t_{1}, \ldots, t_{k}\right) \in X^{k}$ and $\|\mathbf{T}\|_{k}=d$. If $\mathbf{X} \neq \mathbf{0}$ or $\mathbf{Y} \neq \mathbf{0}$, then let

$$
\mathbf{T}= \begin{cases}\left(1+\frac{d}{\|\mathbf{X}\|_{k}}\right) \mathbf{X}, & \|\mathbf{X}\|_{k} \geq\|\mathbf{Y}\|_{k} ; \\ \left(1+\frac{d}{\|\mathbf{Y}\|_{k}}\right) \mathbf{Y}, & \|\mathbf{X}\|_{k}<\|\mathbf{Y}\|_{k} .\end{cases}
$$

If $\|\mathbf{X}\|_{k} \geq\|\mathbf{Y}\|_{k}$, then $\|\mathbf{T}\|_{k}=\|\mathbf{X}\|_{k}+d>d$. If $\|\mathbf{X}\|_{k}<\|\mathbf{Y}\|_{k}$, then $\|\mathbf{T}\|_{k}=\|\mathbf{Y}\|_{k}+d>d$. Therefore,

$$
\begin{aligned}
\|\mathbf{X}-\mathbf{T}\|_{k}+\|\mathbf{Y}+\mathbf{T}\|_{k} & \geq 2\|\mathbf{T}\|_{k}-\left(\|\mathbf{X}\|_{k}+\|\mathbf{Y}\|_{k}\right) \geq d ; \\
\|\mathbf{X}-\mathbf{T}\|_{k}+\|\mathbf{Y}-\mathbf{T}\|_{k} & \geq 2\|\mathbf{T}\|_{k}-\left(\|\mathbf{X}\|_{k}+\|\mathbf{Y}\|_{k}\right) \geq d ; \\
\|\mathbf{X}-2 \mathbf{T}\|_{k}+\|\mathbf{Y}\|_{k} & \geq 2\|\mathbf{T}\|_{k}-\left(\|\mathbf{X}\|_{k}+\|\mathbf{Y}\|_{k}\right) \geq d ; \\
\|\mathbf{T}\|_{k}+\|\mathbf{Y}\|_{k} & \geq d ; \\
\|\mathbf{T}-\mathbf{Y}\|_{k} & \geq d ; \\
\|\mathbf{T}+\mathbf{Y}\|_{k} & \geq d .
\end{aligned}
$$

It follows from (41) that

$$
\begin{aligned}
& \|\left(f\left(x_{1}+y_{1}\right)-f\left(x_{1}-y_{1}\right)\right. \\
& \quad+2 f\left(-y_{1}\right), \ldots, f\left(x_{k}+y_{k}\right)-f\left(x_{k}-y_{k}\right) \\
& \left.\quad+2 f\left(-y_{k}\right)\right)\left\|_{k} \leq\right\|\left(f\left(x_{1}+y_{1}\right)\right. \\
& \quad-f\left(x_{1}-y_{1}-2 t_{1}\right) \\
& \quad+2 f\left(-\left(y_{1}+t_{1}\right)\right), \ldots, f\left(x_{k}+y_{k}\right) \\
& \left.\quad-f\left(x_{k}-y_{k}-2 t_{k}\right)+2 f\left(-\left(y_{k}+t_{k}\right)\right)\right) \|_{k} \\
& \quad+\|\left(f\left(x_{1}+y_{1}-2 t_{1}\right)-f\left(x_{1}-y_{1}\right)\right.
\end{aligned}
$$

$$
\begin{aligned}
& +2 f\left(-\left(y_{1}-t_{1}\right)\right), \ldots, f\left(x_{k}+y_{k}-2 t_{k}\right) \\
& \left.-f\left(x_{k}-y_{k}\right)+2 f\left(-\left(y_{k}-t_{k}\right)\right)\right) \|_{k} \\
& +\|\left(f\left(x_{1}+y_{1}-2 t_{1}\right)-f\left(x_{1}-y_{1}-2 t_{1}\right)\right. \\
& +2 f\left(-y_{1}\right), \ldots, f\left(x_{k}+y_{k}-2 t_{k}\right) \\
& \left.-f\left(x_{k}-y_{k}-2 t_{k}\right)+2 f\left(-y_{k}\right)\right) \|_{k} \\
& +\|\left(2 f\left(t_{1}+y_{1}\right)-2 f\left(t_{1}-y_{1}\right)\right. \\
& +4 f\left(-y_{1}\right), \ldots, 2 f\left(t_{k}+y_{k}\right)-2 f\left(t_{k}-y_{k}\right) \\
& \left.+4 f\left(-y_{k}\right)\right)\left\|_{k}+\right\|\left(2 f\left(t_{1}+y_{1}\right)\right. \\
& -2 f\left(-\left(t_{1}+y_{1}\right)\right), \ldots, 2 f\left(t_{k}+y_{k}\right) \\
& \left.+2 f\left(-\left(t_{k}-y_{k}\right)\right)\right) \|_{k} \leq 7 \delta, \\
& \left\|\left(2 f\left(z_{1}\right)+2 f\left(-z_{1}\right), \ldots, 2 f\left(z_{k}\right)+2 f\left(-z_{k}\right)\right)\right\|_{k} \\
& \quad \leq \|\left(2 f\left(z_{1}\right)+f\left(-z_{1}+t_{1}\right)\right. \\
& -f\left(z_{1}+t_{1}\right), \ldots, 2 f\left(z_{k}\right)+f\left(-z_{k}+t_{k}\right) \\
& \left.-f\left(z_{k}+t_{k}\right)\right)\left\|_{k}+\right\|\left(2 f\left(-z_{1}\right)+f\left(z_{1}+t_{1}\right)\right. \\
& -
\end{aligned}
$$

According to Theorem 10, there exists unique alternative additive mapping of the second form $A: X \rightarrow Y$ such that (42) holds true.

Corollary 12. Let $\left(\left(X^{n},\|\cdot\|\right): n \in N\right)$ be a real multinormed space and let $\left(\left(Y^{n},\|\cdot\|\right): n \in N\right)$ be a multiBanach space. Mapping $f: X \rightarrow Y$ satisfies alternative additive equation of the second form if and only if, for each $k \in N$ if $\left\|\left(x_{1}, \ldots, x_{k}\right)\right\|_{k}+\left\|\left(y_{1}, \ldots, y_{k}\right)\right\|_{k} \rightarrow \infty$ and $\left\|\left(z_{1}, \ldots, z_{k}\right)\right\|_{k} \rightarrow \infty$, one has

$$
\begin{aligned}
& \|\left(f\left(x_{1}+y_{1}\right)-f\left(x_{1}-y_{1}\right)\right. \\
& \quad+2 f\left(-y_{1}\right), \ldots, f\left(x_{k}+y_{k}\right)-f\left(x_{k}-y_{k}\right) \\
& \left.\quad+2 f\left(-y_{k}\right)\right) \|_{k} \longrightarrow 0 ; \\
& \left\|\left(f\left(z_{1}\right)+f\left(-z_{1}\right), \ldots, f\left(z_{k}\right)+f\left(-z_{k}\right)\right)\right\|_{k} \longrightarrow 0 .
\end{aligned}
$$

\section{Competing Interests}

The authors declare that they have no competing interests.

\section{Authors' Contributions}

All authors conceived of the study, participated its design and coordination, drafted the paper, participated in the sequence alignment, and read and approved the final paper. 


\section{Acknowledgments}

This work is supported by the National Natural Science Foundation of China (Grant 11371119), the Key Foundation of Education Department of Hebei Province (Grant ZD2016023), and Natural Science Foundation of Education Department of Hebei Province (Grant Z2014031).

\section{References}

[1] S. M. Ulam, Problems in Modern Mathematics, Wiley, New York, NY, USA, 1960.

[2] D. H. Hyers, "On the stability of the linear functional equation," Proceedings of the National Academy of Sciences of the United States of America, vol. 27, no. 4, pp. 222-224, 1941.

[3] T. M. Rassias, "On the stability of the linear mapping in Banach spaces," Proceedings of the American Mathematical Society, vol. 72, no. 2, pp. 297-300, 1978.

[4] R. P. Agarwal, B. Xu, and W. Zhang, "Stability of functional equations in single variable," Journal of Mathematical Analysis and Applications, vol. 288, no. 2, pp. 852-869, 2003.

[5] Z. Gajda, "On stability of additive mappings," International Journal of Mathematics and Mathematical Sciences, vol. 14, no. 3, pp. 431-434, 1991.

[6] P. Gavruta, "A generalization of the Hyers-Ulam-Rassias stability of approximately additive mappings," Journal of Mathematical Analysis and Applications, vol. 184, no. 3, pp. 431-436, 1994.

[7] S.-M. Jung and J. M. Rassias, "A fixed point approach to the stability of a functional equation of the spiral of Theodorus," Fixed Point Theory and Applications, vol. 2008, Article ID 945010, 7 pages, 2008.

[8] C. Park and J. M. Rassias, "Stability of the Jensen-type functional equation in $C^{*}$-algebras: a fixed point approach", Abstract and Applied Analysis, vol. 2009, Article ID 360432, 17 pages, 2009.

[9] B. Xu and J. Brzdek, "Hyers-Ulam stability of a system of first order linear recurrences with constant coefficients," Discrete Dynamics in Nature and Society, vol. 2015, Article ID 269356, 5 pages, 2015.

[10] B. Xu, J. Brzdęk, and W. Zhang, "Fixed-point results and the Hyers-Ulam stability of linear equations of higher orders," Pacific Journal of Mathematics, vol. 273, no. 2, pp. 483-498, 2015.

[11] X. Yang, L. Chang, G. Liu, and G. Shen, "Stability of functional equations in $(\mathrm{n} ; \beta)$-normed spaces," Journal of Inequalities and Applications, vol. 2015, no. 112, 18 pages, 2015.

[12] X. Zhao, X. Yang, and C.-T. Pang, "Solution and stability of a general mixed type cubic and quartic functional equation," Journal of Function Spaces and Applications, vol. 2013, Article ID 673810, 8 pages, 2013.

[13] N. Brillouët-Belluot, J. Brzdęk, and K. Ciepliński, "On some recent developments in Ulam's type stability," Abstract and Applied Analysis, vol. 2012, Article ID 716936, 41 pages, 2012.

[14] J. Brzdęk, W. Fechner, M. S. Moslehian, and J. Sikorska, "Recent developments of the conditional stability of the homomorphism equation," Banach Journal of Mathematical Analysis, vol. 9, no. 3, pp. 278-326, 2015.

[15] D. H. Hyers, G. Isac, and Th. M. Rassias, Stability of Functional Equations in Several Variables, Birkhäuser, Basel, Switzerland, 1998.

[16] S. Czerwik, Functional Equations and Inequalities in Several Variables, World Scientific, Hackensack, NJ, USA, 2002.
[17] P. Kannappan, Functional Equations and Inequalities with Applications, Springer Monographs in Mathematics, Springer, New York, NY, USA, 2009.

[18] S.-M. Jung, Hyers-Ulam-Rassias Stability of Functional Equations in Nonlinear Analysis, vol. 48 of Springer Optimization and Its Applications, Springer, New York, NY, USA, 2011.

[19] H. G. Dales and M. E. Polyakov, "Multi-normed spaces and multi-Banach algebras".

[20] H. G. Dales and M. S. Moslehian, "Stability of mappings on multi-normed spaces," Glasgow Mathematical Journal, vol. 49, no. 2, pp. 321-332, 2007.

[21] M. S. Moslehian, "Superstability of higher derivations in multiBanach algebras," Tamsui Oxford Journal of Mathematical Sciences, vol. 24, no. 4, pp. 417-427, 2008.

[22] M. S. Moslehian, K. Nikodem, and D. Popa, "Asymptotic aspect of the quadratic functional equation in multi-normed spaces," Journal of Mathematical Analysis and Applications, vol. 355, no. 2, pp. 717-724, 2009.

[23] L. Wang, B. Liu, and R. Bai, "Stability of a mixed type functional equation on multi-Banach spaces: a fixed point approach," Fixed Point Theory and Applications, vol. 2010, Article ID 283827, 9 pages, 2010.

[24] X. Yang, L. Chang, and G. Liu, "Orthogonal stability of mixed additive-quadratic Jensen type functional equation in multiBanach spaces," Advances in Pure Mathematics, vol. 5, no. 6, pp. 325-332, 2015.

[25] J. M. Rassias and M. J. Rassias, "Asymptotic behavior of alternative JENsen and JENsen type functional equations," Bulletin des Sciences Mathématiques, vol. 129, no. 7, pp. 545-558, 2005. 


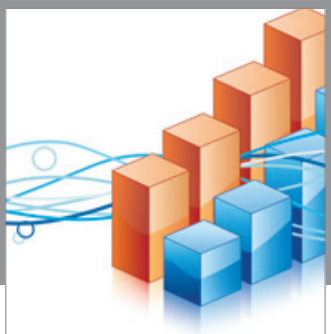

Advances in

Operations Research

vatem alat4

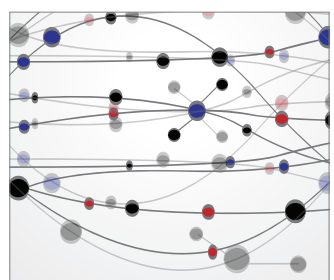

\section{The Scientific} World Journal
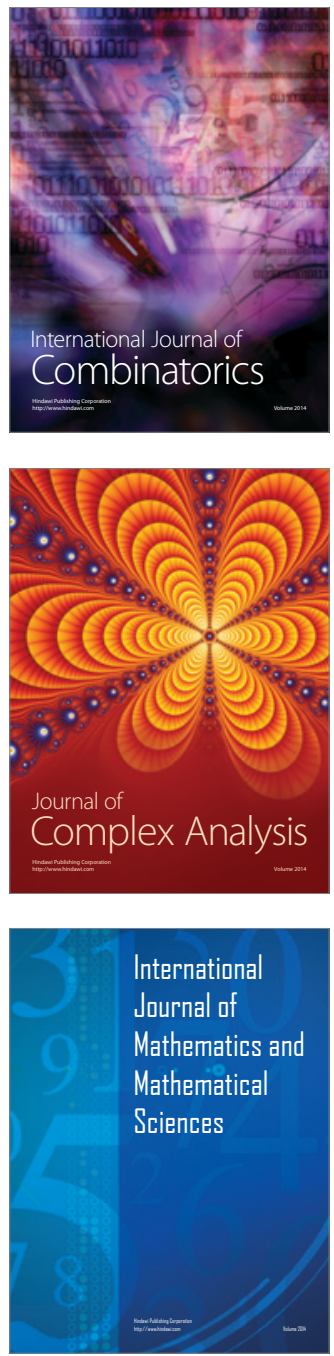
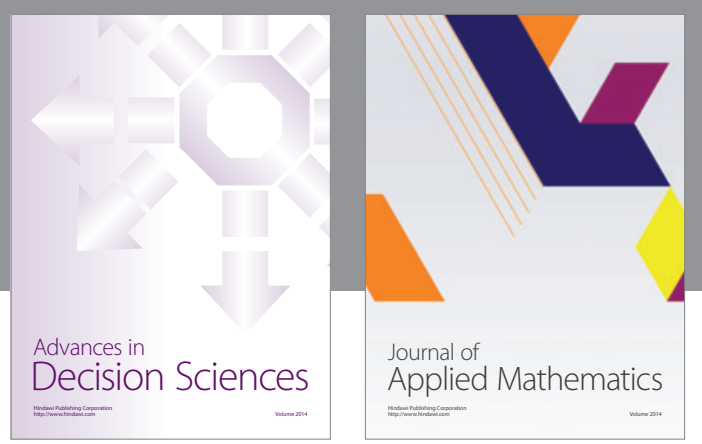

Algebra

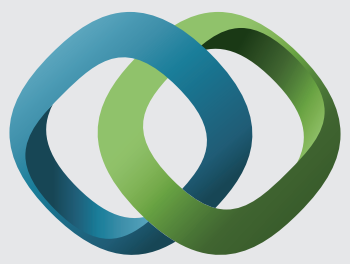

\section{Hindawi}

Submit your manuscripts at

http://www.hindawi.com
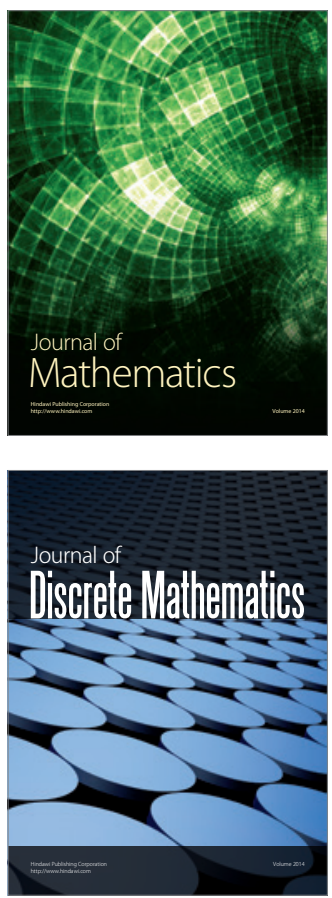

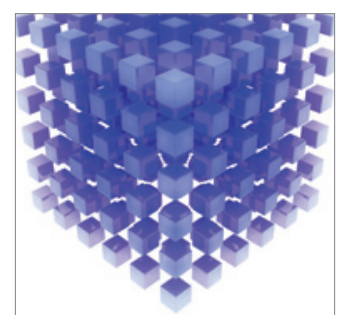

Mathematical Problems in Engineering
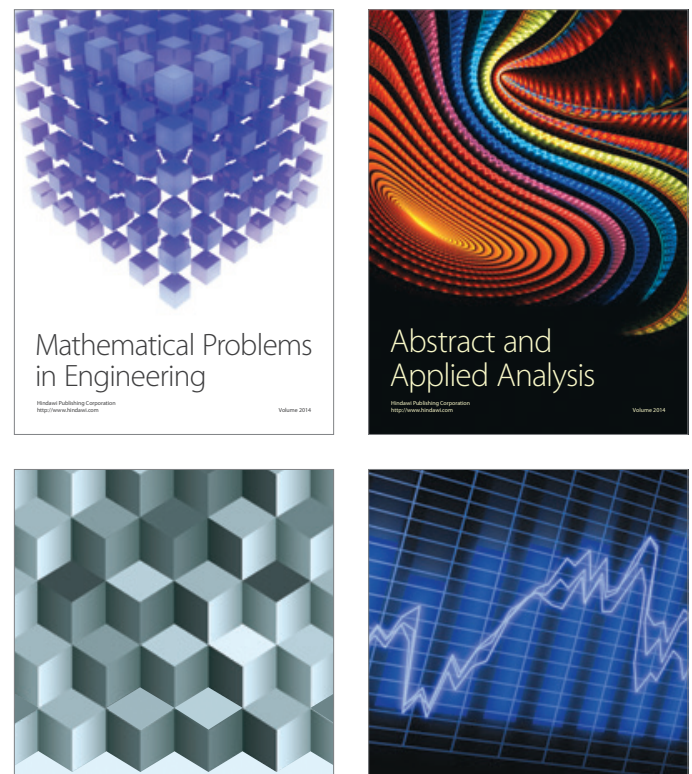

Journal of

Function Spaces

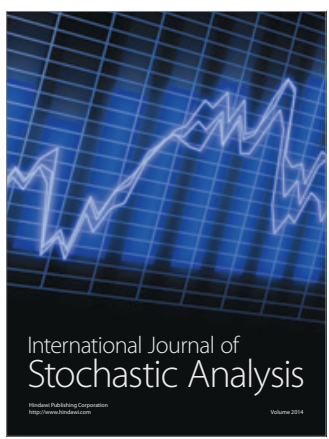

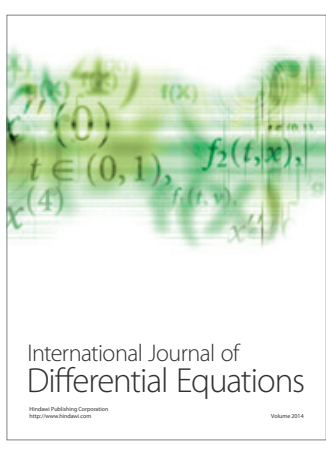
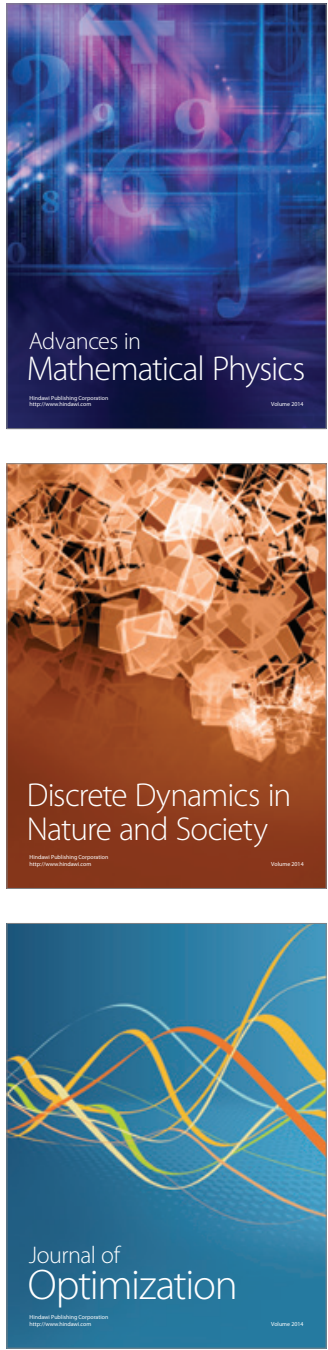